\section{Is pelvic organ prolapse correction with vaginal mesh suitable with a correct indication and protocolized follow-up?}

Pau Sarrio-Sanz, ${ }^{1}$ Ana Isabel LopezLopez, ${ }^{1}$ Laura Martinez-Cayuelas, ${ }^{1}$ Luis Gomez-Perez, ${ }^{2,3}$ Manuel Angel Ortiz-Gorraiz, ${ }^{1}$ Jesus Romero-Maroto ${ }^{1,3}$

${ }^{1}$ Urology Services, University Hospital of San Juan de Alicante, San Juan de Alicante; ${ }^{2}$ Urology Services, General University Hospital of Elche; ${ }^{3}$ Department of Clinical Medicine, Miguel Hernández University, San Juan de Alicante, Spain

\begin{abstract}
The use of vaginal mesh in order to correct Pelvic Organ Prolapse (POP) has been banned by the FDA due to the complications associated with them. The objective is to determine efficacy and safety in the short and long term in a sample of women undergoing transvaginal mesh surgery performed by properly trained surgeons, in a referral center and with a protocolized follow-up. We present a longitudinal, descriptive study of a cohort of 53 patients with POP who underwent transvaginal mesh surgery between 2001 and 2015. The efficacy of the treatment is evaluated quantifying both clinical changes and life quality, as well as the rate for prolapse recurrence and the short-and long-term treatment-related complications. A total of 53 patients with average follow-up of 87 months were included. All of them had their surgery performed by three properly-trained surgeons. Indication for mesh placement was assessed in $49.1 \%$ of cases due to previous surgery recurrence. Treatment improved urinary incontinence rates, constipation, voiding difficulty, dyspareunia and quality of life. Long-term complication rate was $9.6 \%(5.7 \%$ exposure, $1.9 \%$ urinary obstruction and $1.9 \%$ pain). None of the patients presented recurrence in the mesh-treated compartment and 6 patients $(11.3 \%)$ needed surgery after recurrence in a different compartment at the end of follow-up. Vaginal placement of synthetic mesh for POP treatment is safe in the short-, medium- and long-term when performed in referral centers. The correct indication and long-term follow-up are essential to diagnose and treat possible complications.
\end{abstract}

\section{Introduction}

Pelvic Organ Prolapse (POP) is a common condition affecting up to a $50 \%$ of women during their life. ${ }^{1}$ Although it can appear at any age, prolapse-related symptoms most commonly show during the menopausial transition. ${ }^{2}$

Increased life expectancy means that women in developed countries live almost a third of their lives in menopause. This, along with the fact that women lead more active lives, involves an increase in demand for treatment solutions to POP, as it gravely affects women's quality of life and shows an increase in its prevalence. ${ }^{3}$ Diagnose and treatment of POP produces both suffering to the patient and a major healthcare costs, ${ }^{4}$ so it is crucial to optimize the resources in order to offer an effective therapy with the lowest reinterventions and associated complications rate.

The abdominal route (open or laparoscopic) is considered to be the best choice for treating vaginal vault prolapse, proving low recurrence figures, less dyspareunia and vaginal shortening at the expense of higher morbidity, longer surgical time and higher costs.

Traditional techniques with native tissue for vaginal surgery, hysterectomy and colporrhaphy are still a valid option ${ }^{6}$ but there are some circumstances in which they may not be resolutive.

Vaginal mesh placement to correct POP has thrown up great controversy in recent years due to the great number of complications communicated in different countries. ${ }^{7,8}$ Finally, the FDA banned the use of such devices in 2019

However, many series ${ }^{9-14}$ have communicated positive results after using vaginal meshes, therefore the analysis of the factors involved in their appearance gains special relevance.

The main objective of the present work is to evaluate the results obtained, in terms of safety and efficacy, after treating POP with transvaginal mesh surgery in a sample of women operated by properly trained surgeons, in a referral center and with a protocolized follow-up.

\section{Materials and Methods}

We performed a longitudinal, descriptive study of a cohort of patients with POP who underwent transvaginal braidedpolypropylene-mesh $\left(40 \mathrm{~g} / \mathrm{m}^{2}\right)$ surgery between 2001 and 2015. They were all diagnosed, operated and followed-up by the same surgeons (RMJ, GPL, LLA).

The study was approved by a local
Correspondence: Ana Isabel Lopez-Lopez, Urology Services, University Hospital of San Juan de Alicante, Carretera Alicante-Valencia N-332 s/n, 03550 San Juan de Alicante, Spain. Tel.: +34 664487934

E-mail: 1lanais@hotmail.com

Key words: Pelvic organ prolapse; vaginal mesh; urinary incontinence; complications; quality of life.

Acknowledgments: The authors thank Rafae Perdomo Devesa for his help in the English version of the text.

Contributions: PSS, AILL: project development, data collection, manuscript writing, LMC: project development, data collection; LGP: project development, manuscript writing and editing; MAOG: manuscript editing. JRM: project development.

Conflict of interest: The authors declare no conflict of interest.

Availability of data and materials: All data generated or analyzed during this study are included in this published article.

Ethics approval and consent to participate The Ethics Committee of Hospital General Universitario de Elche approved this study (Acta 3_2021). The study is conformed with the Helsinki Declaration of 1964, as revised in 2013, concerning human and animal rights All patients participating in this study signed a written informed consent form for participating in this study.

Informed consent: Written informed consent was obtained from a legally authorized representative(s) for anonymized patient information to be published in this article.

Received for publication: 12 November 2021. Revision received: 10 January 2022

Accepted for publication: 25 January 2022

This work is licensed under a Creative Commons Attribution NonCommercial 4.0 License (CC BY-NC 4.0)

(C) Copyright: the Author(s), 2022

Licensee PAGEPress, Italy

Urogynaecologia 2022; 34:280

doi:10.4081/uij.2022.280

ethics committee. The guidelines of the STROBE statement were followed. ${ }^{15}$

We recorded demographic data, obstetric and surgical history, prolapse-affected vaginal compartment and grade according to Baden and Walker and POP-Q classifications using standardized terminology ICSIUGA. ${ }^{16}$

A POP-related symptoms, including 
sense of bulging in the vagina, urinary incontinence, voiding difficulty, constipation and dyspareunia before and after the surgery, was conducted by medical history. Postoperative visits were scheduled a month, six months, one, two, five and ten years after the surgery. Patients with less than one-year follow-up were excluded. Patients who had lost follow-up were contacted by telephone and all those who were still alive accepted another visit. At every visit, $250 \mathrm{~mL}$ of saline was instilled into the bladder using a female bladder catheter. After its removal, urinary incontinence and prolapse exploration was conducted and then the patient underwent an uroflowmetry.

Urinary incontinence was preoperatively classified on four different types: stress, occult or latent stress incontinence, urgency and mixed. The impact of incontinence and POP on quality of life was evaluated by validated questionnaires in Spanish; IQOL, ${ }^{17}$ ICQ-SF, ${ }^{18}$ PGIS, ${ }^{19}$ SUIQ $^{19}$ and PISQ- $12,{ }^{20}$ that were fulfilled before and each appointment after the surgery, and PGII ${ }^{21}$ after it. Questionnaire PISQ-12 was used to evaluate if the patient was sexually active or not and if dyspareunia was present. The postoperative questionnaires results are from the last appointment.

Prolapse surgical correction with mesh was indicated for patients with symptomatic prolapse stage II and higher and affected by previous surgery recurrence or for young patients with anterior compartment POP and without central compartment POP. Patients with apical POP were treat with an abdominal colposacropexy.

Antibiotic prophylaxis was administered during surgery and every effort was made in order to minimize both hematoma and mesh frowning.

We used the Pinnacle Pelvic Floor Repair Kit on anterior and posterior cases, Uphold kit on anterior POP or self-prepared mesh before disposing it, depending on the surgeons' criteria. Hydrodissection was made with adrenaline solution and a vertical incision was performed in every case. Depending on anterior or posterior POP, bladder or rectum was widely dissected from vaginal wall. Every mesh was fixed posteriorly to the sacrospinous ligament. Anteriorly, the cystocele mesh was fixed to the tendinous arch and the rectocele mesh to the puborectalis fascia and perineal body. Two anchoring sutures from the mesh to the cervix were performed in case of any grade of uterine prolapse. Patients with apical POP were treat with an abdominal colposacropexy. The vaginal wall was not trimmed in any patient. A vesical catheter and a vaginal tamponade were maintained during the first 48 hours.

A Transobturator Adjustable Mesh (TOA) was placed in those patients suffering from either preoperative stress or stresspredominant mixed urinary incontinence. ${ }^{22}$

Anatomical cure was defined both as the absence of prolapse and as having a stage II or lower prolapse in the operated compartment. Clinical cure was defined as the disappearance of the sense of bulging in the vagina associated with the anatomical cure. Surgery was considered to be successful when answers "very much better", "much better" or "a little better" in the PGII questionnaire ${ }^{21}$ postoperatively fulfilled by the patient were associated with both anatomical and clinical cure. These rates were evaluated within 6 months, 1, 2, 5 and 10 years

Recurrence was defined as the presence of a symptomatic POP within six months after surgery. De novo dyspareunia was revealed in patients with no dyspareunia before surgery and with prevalence of dyspareunia one year after the surgery.

Early complications (first 30 days) were classified according to the Clavien-Dindo grading system ${ }^{23}$ and late complications according to the ICS-IUGA system. ${ }^{24}$

Descriptive and analytical statistics were performed in SPSS software version 25. For continuous variables, the average was calculated when normal distribution or the median and the interquartile range when non-normal. Quantitative variables were compared by using the chi-square test.

Table 1. Sociodemographic variables and preoperative clinical parameters.

\begin{tabular}{lc}
\hline Age (years) & 64 \\
Smoker or ex-smoker & 13.2 \\
\hline Number of vaginal deliveries (n)* & 2 \\
Instrumental delivery & 17.3 \\
\hline Menopausal patients & 94.2 \\
Sense of bulging in the vagina & 86.7 \\
\hline Voiding difficulty & 26 \\
Constipation & 17 \\
\hline Dyspareunia** & 12 \\
Previous plastic surgery & 49.1 \\
\hline Previous hysterectomy & 52.8 \\
Previous UI surgery & 15.3 \\
\hline Urinary incontinence (UI) & 66 \\
Stress UI & 11.5 \\
\hline Stress occult UI & 11.5 \\
Mixed UI & 26.9 \\
\hline Urge UI & 17.3 \\
\hline *The medin is caluted ** Pationts with dysparenin (59.5\% of the sample).
\end{tabular}

\footnotetext{
* The median is calculated. ** Patients with dyspareunia ( $59.5 \%$ of the sample).
}

\section{Results}

A total of 53 patients were included with an average follow-up of 87 months (range 12-215). Demographic characteristics and preoperative clinical parameters are described in Table 1.

A mesh was placed in the anterior vaginal compartment in $73.6 \%(n=39)$ of the patients, in the posterior compartment in $(n=7)$. A sling for urinary incontinence correction was placed in the same surgical procedure in $45.3 \%(n=23)$ of the patients. Of them, an anterior vaginal mesh had been placed in 18 of them (78.2\%), a posterior vaginal mesh in 2 of them $(8.7 \%)$ and both anterior and posterior in 3 of them (13\%).

The evolution of the anatomical, clinical and surgery-success rates are shown in Figure 1.

Postoperative clinical changes related to the sense of bulging in the vagina, voiding difficulty, constipation and dyspareunia are summarized in Table 2.

The answers to the questionnaires are shown in Table 3.

The response rate to the questionnaire PGII was $78.8 \% \quad(n=41) .68 .2 \%$ of the patients answered feeling "very much better" or "much better" and $21.9 \%$ "a little better".

There was one $(1.9 \%)$ early complication and it was a bladder perforation. Through the follow-up, complications were noted in 5 patients $(9.6 \%)$; 3 patients $(5.7 \%)$ presented vaginal mesh exposure. All of them were surgically solved. One patient $13.2 \%(n=7)$ and in both of them in $13.2 \%$ 
Table 2. Clinical evolution of the symptoms aforementioned by the patients depending on the type of mesh used.

\begin{tabular}{|c|c|c|c|c|c|c|c|c|c|}
\hline & \multicolumn{3}{|c|}{ Anterior MESH } & \multicolumn{3}{|c|}{ Posterior MESH } & \multicolumn{3}{|c|}{ Anterior + Posterior MESH } \\
\hline & Preop & $1 y$ & $5 y$ & Preop & $1 \mathrm{y}$ & $5 y$ & Preop & ly & $5 y$ \\
\hline $\mathrm{N}$ & 39 & 36 & 21 & 7 & 5 & 3 & 7 & 4 & 2 \\
\hline Sense of bulging in the vagina (\%) & 87.1 & 0 & 14.2 & 71.4 & 0 & 0 & 85.7 & 25 & 0 \\
\hline Voiding difficulty (\%) & 28.2 & 15.4 & 9.5 & 14.3 & 20 & 0 & 28.6 & 0 & 0 \\
\hline Constipation (\%) & 10.3 & 11.3 & 9.5 & 57.2 & 20 & 0 & 14.3 & 0 & 0 \\
\hline Active sexuality & 64.1 & 55 & 62 & 57 & 60 & 66 & 42 & 50 & 50 \\
\hline Dyspareunia* (\%) & 9.5 & 6.6 & 0 & 0 & 0 & 0 & 0 & 0 & 0 \\
\hline
\end{tabular}

*For the analysis of the ratio of patients with dyspareunia, only those with an active sexuality are considered. Preop: preoperative value. ly: one year. 5y: five years.

$(1.9 \%)$ underwent removal of part of the mesh due to focused pain in the sacral region refractory to medical treatment and another patient $(1.9 \%)$ due to urinary obstruction (Table 4). The median time to reoperation was 53 months (RI 25-60 months).

None of the patients required surgery for recurrence in the same compartment in which the transvaginal mesh had been placed. At the end of the follow-up, 6 patients $(11.3 \%)$ required new surgery due to recurrence in a different compartment (Table 4).

On the flowmetry, the preoperative average maximum flow was $16.4 \mathrm{ml} / \mathrm{min}$. After the surgery the average maximum flow was $17.48 \mathrm{~mL} / \mathrm{min}$ at 6 months, 15.9 $\mathrm{mL} / \mathrm{min}$ one year after, 16.6 after two years and 15.8 at 5 years $(\mathrm{p}=0,62)$.

$49.1 \%$ of patients $(n=26)$ had a previous surgery to treat their POP. The mean age in this subgroup is 65.3 years. $11.5 \%(n=3)$ had complications related to the mesh implant (similar to the entire cohort) and $15.3 \%(n=4)$ had a recurrence, on average at two years (Table 4).

\section{Discussion}

The FDA ban on the use of vaginal mesh in order to correct pelvic organ prolapse has meant a therapeutic change to the protocols of many Pelvic Floor Units around the world. While it is true that the recent published literature about complications related to the use of vaginal mesh was worrisome to all the urogynecologists, many of them have witnessed its publication without finding their patients represented on the published data.

The results obtained in the present study in terms of effectiveness are comparable to the data published in the literature (Table 5). ${ }^{10,11,25}$

Although the results obtained in our study regarding anatomical cure rates are encouraging (it reaches $96 \%$ after a year

Table 3. Answers to the questionnaires.

\begin{tabular}{lccc} 
Questionnaires & Prior to surgery & After surgery & P \\
IQOL [13] & 60.4 & 84.24 & 0.269 \\
ICIQ-SF [14] & 15.71 & 7.87 & 0.407 \\
\hline SUIQ [15] & 14.75 & 3.08 & 0.047 \\
PGIS [15]: none+mild & $24 \%$ & $76 \%$ & 0.01 \\
\hline PGIS [15]: moderate+severe & $76 \%$ & $24 \%$ & 0.01 \\
\hline
\end{tabular}

IQOL: incontinence quality of life (range 0 to 100); ICIQ-SF: questionnaire about how incontinence affects quality of life; SUIQ: number of incontinence episodes within 7 days; PGIS: patient global impression of severity.

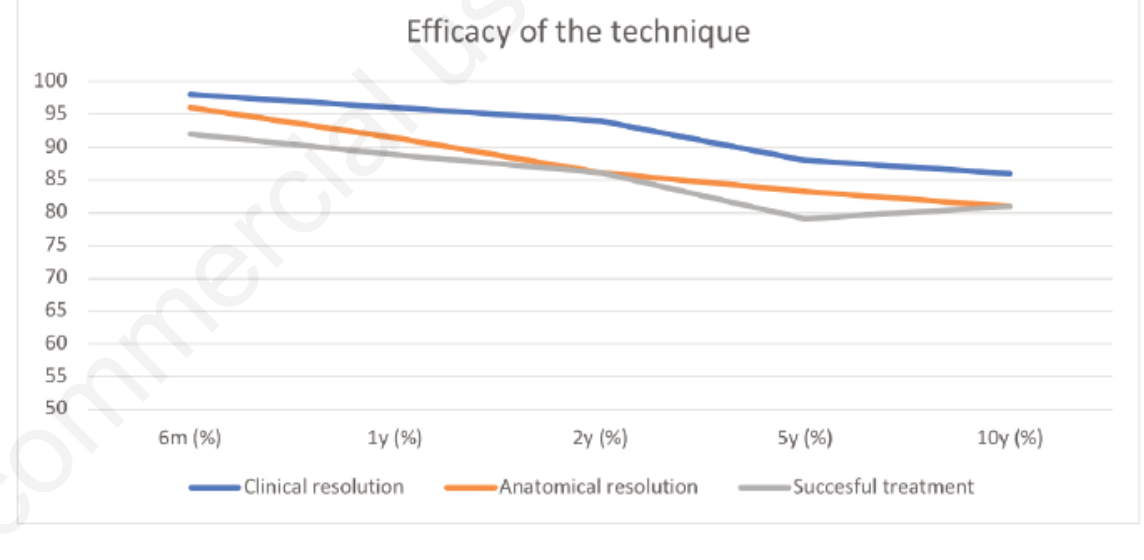

Figure 1. Evolution of the anatomical cure rate, clinical cure rate and success rate.

and then steadily decreases to $81 \%$ after ten years), in the authors' view, both those regarding clinical cure rates are $(96 \%$ after a year and $86 \%$ after ten years) and specially those regarding surgery success rates (anatomical cure + clinical cure + patient subjective evaluation, which reaches $86 \%$ after a year and $81 \%$ after ten years) are of most interest, due to the fact that the disappearance of symptoms is the primary objective of POP treatment.

As can be seen in Table 1, half of the patients operated during this study had already underwent at least one previous unsuccessful POP-correcting vaginal surgery, none of which with vaginal mesh. These are patients treated into routine clinical practice, with previous surgeries and in half of the cases, with incontinence requir- ing surgical treatment. These characteristics are considered in some published literature as exclusion criteria. ${ }^{25-29}$

From among the evaluated clinical parameters, the disappearance of the sense of bulging in the vagina showed the best result. Voiding difficulty showed a significant improvement in those patients with anterior compartment surgery. Constipation improved fundamentally in patients with posterior compartment surgery, given that constipation is not that frequent in patients with affected anterior and middle compartments. On an overall basis, almost $50 \%$ of the cases with previous voiding difficulty improved after POP surgery. Regarding voiding syndrome symptoms: improvement of the urine flow, decrease of the diurnal and nocturnal frequency and decrease of the 
need for special body positions to make urination easier or even the prolapse manual reduction (data not published in this study).

Regarding intra-operative complications, our $1.9 \%$ reported rate is within the parameters reported in the revised series for the injury of nearby organs. ${ }^{26}$

Regarding dyspareunia, $53 \%$ of our patients were sexually active before the surgery, a higher figure than the one presented in the literature ${ }^{26,28}$ and similar to the one presented by Gonzalez-Palanca et al. ${ }^{10}$ Sexual activity remained stable along the follow-up. The reasons for preoperative sexual abstinence given by the patients were widowhood or partner absence, followed by low libido or partner's erection difficulties.
De novo dyspareunia after vaginal mesh surgery thoroughly varies depending on the consulted literature $(0-14 \%) .{ }^{10,30}$ No case of de novo dyspareunia after surgery was reported in our series, but the percentage of women showing pain or discomfort during the sexual intercourse amounted to $21 \%$ after six months (compared to the previous $9.5 \%$ ) and then, decreased to $6.6 \%$ in the 12 -month visit. The reasons for dyspareunia in women are heterogeneous and difficult to evaluate, including vaginal length, and having a partner. ${ }^{31}$ This initial worsening of dyspareunia may be due to the tissular scarring phenomena entailed by the implementation of synthetic materials in menopausal patients with fragile vaginal tissue. On a Cochrane meta-analysis published in 2016,
11 randomized clinical trials which included 764 patients were analyzed, without revealing any statistically significant difference between de novo dyspareunia in women whose surgery included vaginal mesh placement and in those whose surgery implied the use of native tissues $(\mathrm{RR}=0.92$, $0.58-1.47) .^{32}$

It has been described in randomized studies a greater immediate post-operative pain in patients whose surgery included vaginal mesh placement than in those whose surgery implied the use of native tissues. ${ }^{29}$ In our experience, these patients needed more analgesia during hospitalization compared to the patients who had a hysterectomy or a vaginal colporrhaphy in our department.

Table 4. Description the patients with early, late complications (Clavien-Dindo, ICS-IUGA) ${ }^{19,20}$ and recurrences.

\begin{tabular}{|c|c|c|c|c|c|c|c|}
\hline \multicolumn{2}{|c|}{ Patient numberAge } & $\begin{array}{c}\text { POP previous } \\
\text { Surgeries }\end{array}$ & Type of mesh & Complications & $\begin{array}{l}\text { Months until } \\
\text { complication }\end{array}$ & Treatment & ICS-iuga / c-d \\
\hline 1 & 67 & No & Anterior + TOA & $\begin{array}{c}\text { Bladder } \\
\text { perforation }\end{array}$ & Intraoperative & Repair & 4AT1S3 / C-D III \\
\hline 2 & 53 & No & Anterior & Voiding difficulty & 3 & Partial mesh removal & 1AcT3SO \\
\hline 3 & 69 & Yes $(\mathrm{H}+\mathrm{C})$ & Anterior & Chronic pelvic pain & 25 & Partial mesh removal & 6BeT4S4 \\
\hline 4 & 73 & Yes (CA) & Anterior + posterior & Posterior mesh exposure & 53 & Surgical repair & 1BbT4S2 \\
\hline 5 & 56 & Yes (CA) & Anterior + TOA & Anterior mesh exposure & 60 & Surgical repair & 3AaT4S1 \\
\hline 6 & 80 & No & Anterior + posterior + TOA & Anterior mesh exposure & 81 & Surgical repair & 2AaT4S2 \\
\hline 1 & 80 & No & Anterior + Posterior + TOA & Uterus IV & 12 & Hysterectomy + colposacropexy & \\
\hline 2 & 64 & Yes $(\mathrm{H}+\mathrm{C})$ & Anterior + posterior & Vaginal vault III & 3 & Colposacropexy & \\
\hline 3 & 42 & No & Anterior & Rectocele III & 46 & Posterior mesh & \\
\hline 4 & 58 & Yes (CA) & Anterior & Uterus III & 49 & $\begin{array}{l}\text { Vaginal hysterectomy + } \\
\text { posterior colporrhaphy }\end{array}$ & \\
\hline 5 & 53 & Yes $(\mathrm{H}+\mathrm{C})$ & Anterior & Enterorectocele III & 22 & Posterior mesh & \\
\hline 6 & 63 & Yes (CA) & Posterior & Cistocele III & 24 & Anterior mesh & \\
\hline
\end{tabular}

*POP degree according to POP-Q classification. ${ }^{12} \mathrm{C}-\mathrm{D}$ : Clavien-Dindo; H+C: hysterectomy and colporrhaphy; CA: anterior colporrhaphy.

Table 5. Comparison between current series and other series described in literature.

\begin{tabular}{|c|c|c|c|c|c|c|c|c|c|}
\hline & n & $\begin{array}{c}\text { Age } \\
\text { (years) }\end{array}$ & $\begin{array}{c}\text { Previous HT } \\
(\%)\end{array}$ & $\begin{array}{l}\text { Previous } \\
\text { surgery (\%) }\end{array}$ & $\begin{array}{l}\text { Follow-up } \\
\text { (months) }\end{array}$ & $\begin{array}{l}\text { SUI synchronic } \\
\text { sling }\end{array}$ & $\begin{array}{l}\text { Early } \\
\text { comp. }(\%)\end{array}$ & $\begin{array}{c}\text { Exposure } \\
(\%)\end{array}$ & $\begin{array}{c}\text { Reinterventions } \\
(\%)\end{array}$ \\
\hline Vinchant et al. ${ }^{14}$ & 29 & 62 & & $6.3 \%$ & 216 & 35 & 1.6 & 25 & 35 \\
\hline Pécheux et al. ${ }^{11}$ & 349 & 63.9 & 24.4 & 17.7 & 102 & 2.9 & & 2.6 & 14.3 \\
\hline Sarrió et al. & 53 & 64 & 52.8 & 49.1 & 87 & 45.3 & 1.9 & 5.7 & 9.6 \\
\hline Duraes et al. ${ }^{9}$ & 270 & 65.2 & 12.4 & 11.5 & 66 & 17.7 & & 0.4 & 3.4 \\
\hline Laso-Garcia et al..$^{26}$ & 75 & 67.6 & 26.3 & 0 & 64 & 58 & 2.6 & 13 & 8.9 \\
\hline de Landsheere et al..$^{13}$ & 524 & 64 & 21.2 & 18.7 & 38 & 34 & 0.6 & 2.5 & 3.6 \\
\hline Rudnicki et al..$^{27 *}$ & 78 & 64.7 & 7.7 & 0 & 36 & 0 & 11.6 & 14.7 & 6.65 \\
\hline González-Palanca et al..$^{10}$ & 58 & 66.5 & 25.86 & 20.69 & 34 & 6.9 & 9.36 & 1.7 & 1.7 \\
\hline Balchandra et al..$^{12}$ & 159 & 61 & 88 & & 28 & 20 & 11 & 4 & 8.3 \\
\hline Glazener et al. ${ }^{28 *}$ & 435 & 59.5 & 29 & 13 & 24 & 0 & 10 & 12 & 9 \\
\hline Altman et al..$^{29 *}$ & 186 & 64.3 & 23 & 16.5 & 12 & 0 & 10 & 3.2 & 3.2 \\
\hline Stanford et al. ${ }^{25}$ & 142 & 64.7 & 43.8 & 0 & 12 & 0 & & 6.3 & 4.22 \\
\hline Rodrigues et al..$^{30}$ & 97 & 61.2 & 21.8 & 21.8 & 12 & 42.6 & 12.4 & 7.2 & 6.2 \\
\hline
\end{tabular}

Previous surgery: POP surgery previous to the analyzed surgery. SUI: stress urinary incontinence. HT: hysterectomy Early Comp: early complications. * Series in which only data of patients with synthetic mesh implant are included. 
During the follow-up, no pain or analgesia-need was observed in the post-operative visits in $98 \%$ of the patients, similar to previously chronic pain rates published. ${ }^{8}$ One patient showed sacral pain in the 12month visit. After the failure of the conservative treatment with first-and-second-step analgesics, a complete mesh removal was performed 25 months after its placement, improving pain relief.

These results regarding the incidence of dyspareunia and pain are reassuring, given that the studies that analyze the reasons for mesh removal include mainly women that show disabling pelvic or vaginal pain or dyspareunia as symptoms. ${ }^{33}$

The mesh exposure rate varies from $0.48 \%$ to $25 \%{ }^{9,10,14,26,27}$ and the surgical repair rate from $33 \%$ to $100 \%$. Factors related with this complication may be the age of the patients ( 73 and 80 years) at the moment of the exposure and a history of previous surgery. These factors suggest the role of postmenopausal vaginal atrophy with vascularization loss as the main cause for the vaginal mesh exposure..$^{10,25,26}$ The exposure rate is higher when the follow-up is longer, like in our study or in Vinchant et al. ${ }^{14}$

Regarding POP recurrence, the absence of recurrence observed in the mesh-treated compartment needs to be highlighted. The six patients that needed reoperation due to POP, needed it due to a new appearing prolapse in a different compartment, mainly because the middle compartment was affected.

Regarding quality of life, over $90 \%$ of the patients stated feeling "very much better", "much better" and "a little better", according to questionnaire PGII.

There are some limitations of the current study, the retrospective non-randomized design, the fact that it is a single-centre study and the small number of patients. Previous studies published in literature, in all but a few cases, have been retrospective and single-centre. ${ }^{25,27,28}$

Respect the small number of patients treated with a vaginal mesh, the authors find interesting to note that the number of patients who underwent vaginal mesh surgery does not reflect, however, the clinical workload of their unit; but rather it could be considered as the consequence of the precise indication established prior to vaginal mesh surgery. In the daily clinical routine of our centre the number of patients suitable of being treated through traditional vaginal surgery, consisting of hysterectomy and colporrhaphy, is high. Menopausal women with uterine prolapse, associated with cystocele and a certain degree of rectocele, comprise the majority of the patients, and the results that we obtain with this approach are satisfactory, with a recurrence rate under $10 \%$ and an improvement of the quality of life.

The main strength of this study comes from the long and protocoled follow-up that we performed. It has enabled us to diagnose and treat the complications detected, half of which occurred after 53 months.

The clinical experience of the surgeons regarding POP allows to diagnose and to treat the patients via the 3 possible surgical approaches (traditional vaginal, vaginal mesh and colposacropexy, open or laparoscopic). A trained surgeon should have good anatomical knowledge, some practice on cadaver surgery and have performed at least 20 vaginal mesh implants. He should know other approaches to treat POP and should be able to solve surgery complications. Such experience facilitates the indication of the most suitable surgery for each patient.

These results raise the need to develop more prospective and randomized studies in order to evaluate the safety of vaginal mesh in centers with experience enough. In the daily clinical routine, we consider that given our long-term results and the experience of our team, the use of vaginal mesh in order to correct POP should be an option if there is an appropriate indication and always providing the patient with the information about risks and alternatives. Knowing which patients have a higher risk of failure and/or complications (aged, vaginal atrophy), we can avoid vaginal mesh surgery in these cases and keep it for those patients with a better success rate and a lower rate of failure or complications.

\section{Conclusions}

The use of vaginal mesh in order to correct $P O P$ has proven to be a safe technique in the short, medium and long term in women who underwent surgery in a specialized center with trained surgeons and selected cases.

In cases of only anterior compartment prolapse with a recurrence or in those cases with a high preoperative risk of recurrence, the use of transvaginal mesh is a safe alternative. The benefits and complications should be considered in each case separately and discussed with the patient in order to optimize the expectations.

\section{References}

1. Barber MD, Maher C. Epidemiology and outcome assessment of pelvic organ prolapse. Int Urogynecology $\mathrm{J}$
2013;24:1783-90.

2. Weintraub AY, Glinter H, MarcusBraun N. Narrative review of the epidemiology, diagnosis and pathophysiology of pelvic organ prolapse. Int Braz J Urol Off J Braz Soc Urol 2020;46:5-14.

3. Wu JM, Hundley AF, Fulton RG, Myers ER. Forecasting the prevalence of pelvic floor disorders in U.S. Women: 2010 to 2050. Obstet Gynecol 2009; 114:1278-83.

4. Cheon C, Maher C. Economics of pelvic organ prolapse surgery. Int Urogynecology J 2013;24:1873-6.

5. Mowat A, Maher D, Baessler K, et al. Surgery for women with posterior compartment prolapse. Cochrane Database Syst Rev 2018;3:CD012975.

6. Hagen S, Stark D. Conservative prevention and management of pelvic organ prolapse in women. Cochrane Database Syst Rev 2011;CD003882.

7. Chapple CR, Cruz F, Deffieux X, et al. Consensus Statement of the European Urology Association and the European Urogynaecological Association on the Use of Implanted Materials for Treating Pelvic Organ Prolapse and Stress Urinary Incontinence. Eur Urol 2017;72:424-31.

8. Kato K, Suzuki S, Hattori R. Mesh exposure after transvaginal mesh prolapse surgery: Out of permissible range? Int J Urol 2021;28:202-7.

9. Duraes M, Panel L, Cornille A, Courtieu C. Long-term follow-up of patients treated by transvaginal mesh repair for anterior prolapse. Eur J Obstet Gynecol Reprod Biol 2018;230:124-9.

10. González Palanca SJ, González Veiga EJ, Palmeiro Fernández G, et al. Longterm results of genital prolapse surgery with polypropylene mesh. Actas Urol Esp 2019;43:254-61.

11. Pécheux O, Giraudet G, Drumez E, et al. Long-term ( 8.5 years) analysis of the type and rate of reoperation after transvaginal mesh repair (Prolift $($ ) ) in 349 patients. Eur J Obstet Gynecol Reprod Biol 2019;232:33-9.

12. Balchandra P, Marsh F, Landon C. Perioperative outcomes and prospective patient reported outcome measures for transvaginal mesh surgery. Arch Gynecol Obstet 2015;292:875-82.

13. de Landsheere L, Ismail S, Lucot J-P, et al. Surgical intervention after transvaginal Prolift mesh repair: retrospective single-center study including 524 patients with 3 years' median follow-up. Am J Obstet Gynecol 2012;206:83.e17.

14. Vinchant M, Bitumba I, Letouzey V, et 
al. Reoperation rate and outcomes following the placement of polypropylene mesh by the vaginal route for cystocele: very long-term follow-up. Int Urogynecol J 2021;32:929-35.

15. von Elm E, Altman DG, Egger M, et al. The Strengthening the Reporting of Observational Studies in Epidemiology (STROBE) statement: guidelines for reporting observational studies. J Clin Epidemiol 2008;61:344-9.

16. Haylen BT, Maher CF, Barber MD, et al. An International Urogynecological Association (IUGA) / International Continence Society (ICS) joint report on the terminology for female pelvic organ prolapse (POP). Int Urogynecology J 2016;27:165-94.

17. Patrick DL, Martin ML, Bushnell DM, et al. Quality of life of women with urinary incontinence: further development of the incontinence quality of life instrument (I-QOL). Urology 1999;53:71-6.

18. Espuña Pons M, Rebollo Alvarez P, Puig Clota M. [Validation of the Spanish version of the International Consultation on Incontinence Questionnaire-Short Form. A questionnaire for assessing the urinary incontinence]. Med Clin (Barc) 2004;122:28892.

19. Musibay ER, Borges Sandrino RS. Cuestionarios de calidad de vida en las mujeres con disfunciones del suelo pélvico. Rev Cuba Obstet Ginecol 2016;42:372-85.

20. Pons EM, Clota PM, Aguilón GM, et al. [Questionnaire for evaluation of sexual function in women with genital prolapse and/or incontinence. Validation of the Spanish version of 'Pelvic Organ Prolapse/Urinary Incontinence Sexual
Questionnaire (PISQ-12)']. Actas Urol Esp 2008;32:211-9.

21. Srikrishna S, Robinson D, Cardozo L. Validation of the Patient Global Impression of Improvement (PGI-I) for urogenital prolapse. Int Urogynecology J 2010;21:523-8.

22. Romero Maroto J, Ortiz Gorraiz M, Prieto Chaparro L, et al. TVA and TOA. New adjustable mesh for the treatment of female stress incontinence. Preliminaries results. Actas Urol Esp 2006;30:186-94.

23. Dindo D, Demartines N, Clavien P-A. Classification of surgical complications: a new proposal with evaluation in a cohort of 6336 patients and results of a survey. Ann Surg 2004;240:205-13.

24. Haylen BT, Freeman RM, Swift SE, et al. An International Urogynecological Association (IUGA)/International Continence Society (ICS) joint terminology and classification of the complications related directly to the insertion of prostheses (meshes, implants, tapes) and grafts in female pelvic floor surgery. Neurourol Urodyn 2011;30:212 .

25. Stanford EJ, Moore RD, Roovers JPWR, et al. Elevate anterior/apical: 12month data showing safety and efficacy in surgical treatment of pelvic organ prolapse. Female Pelvic Med Reconstr Surg 2013;19:79-83.

26. Laso-García IM, Rodríguez-Cabello MA, Jiménez-Cidre MA, et al. Prospective long-term results, complications and risk factors in pelvic organ prolapse treatment with vaginal mesh. Eur J Obstet Gynecol Reprod Biol 2017;211:62-7.

27. Rudnicki M, Laurikainen E, Pogosean $\mathrm{R}$, et al. A 3-year follow-up after anteri- or colporrhaphy compared with collagen-coated transvaginal mesh for anterior vaginal wall prolapse: a randomised controlled trial. BJOG Int J Obstet Gynaecol 2016;123:136-42.

28. Glazener CM, Breeman S, Elders A, et al. Mesh, graft, or standard repair for women having primary transvaginal anterior or posterior compartment prolapse surgery: two parallel-group, multicentre, randomised, controlled trials (PROSPECT). Lancet Lond Engl 2017;389:381-92.

29. Altman D, Väyrynen T, Engh ME, et al. Nordic Transvaginal Mesh Group. Anterior colporrhaphy versus transvaginal mesh for pelvic-organ prolapse. $\mathrm{N}$ Engl J Med 2011;364:1826-36.

30. Palma PCR, Monteiro MV de C, Ledesma MA, et al. Treatment of anterior vaginal wall prolapse using transvaginal anterior mesh with apical fixation: a prospective multicenter study with up to 2 years of follow-up. Int Neurourol J 2018;22:177-84.

31. Anglès-Acedo S, Ros-Cerro C, EscuraSancho S, et al. Female sexuality before and after sacrocolpopexy or vaginal mesh: is vaginal length one of the key factors? Int Urogynecol J 2022;33:14352.

32. Maher C, Feiner B, Baessler K, et al. Transvaginal mesh or grafts compared with native tissue repair for vaginal prolapse. Cochrane Database Syst Rev 2016;2016.

33. MacDonald S, Terlecki R, Costantini E, Badlani G. complications of transvaginal mesh for pelvic organ prolapse and stress urinary incontinence: tips for prevention, recognition, and management. Eur Urol Focus 2016;2:260-7. 